treatment efforts. OBJECTIVES/GOALS: Given the advantages of ecological momentary assessment (EMA), and the lack of research on non-suicidal self-injury (NSSI) in military populations, the goal of the current pilot study is to determine the feasibility of using EMA to assess daily changes in post-traumatic stress disorder (PTSD) symptoms, negative affect and NSSI in veterans with PTSD. METHODS/STUDY POPULATION: Twenty military veterans with post-traumatic stress disorder (PTSD) who have engaged in non-suicidal self-injury (NSSI) in the previous 12 months will be recruited. Participants will complete assessments 4 times per day for 28 days at randomly scheduled times. Assessments will measure PTSD symptoms, negative emotions, and NSSI urges and behaviors. At the conclusion of the 28-day study period, participants will complete measures that ask about their experiences in the study, (e.g., the acceptability of the daily surveys and the accessibility of the webbased surveys). Feasibility will be determined with regard to the success of recruiting eligible participants and compliance with daily survey completion. Variability in PTSD symptoms, negative affect, and NSSI urges and behaviors also will be determined. RESULTS/ ANTICIPATED RESULTS: It is anticipated that this study will successfully recruit 20 veterans with PTSD with a history of engaging in NSSI within the previous 12 months. It is also anticipated that daily survey completion rates will be approximately $90 \%$ based on previous research using EMA with veterans with PTSD and that participants will indicate satisfaction with the procedures of the study. It is anticipated that participants will demonstrate variability in PTSD symptoms, indicated by changes in the number of symptoms endorsed and the intensity of those symptoms experienced. Finally, it is anticipated that participants will demonstrate variability in negative emotions (fear, hostility, guilt, and sadness).

DISCUSSION/SIGNIFICANCE OF FINDINGS: Findings from this study will support the use of EMA in a subsequent large-scale investigation examining time-varying symptoms of PTSD and negative affect as antecedents to NSSI. Information from this large-scale study will in turn be used to inform treatments that may to decrease NSSI in veterans by targeting specific symptoms and negative emotions.

63230

Quantifying Heavy Metals in Interstitial Fluid for Remote Monitoring of Chronic Exposures

Robert Taylor ${ }^{1}$, Alicia Bolt ${ }^{1}$, Abdulmehdi Ali ${ }^{2}$, Yiliang $\mathrm{Zhu}^{1}$ and

${ }^{*}$ Justin Baca ${ }^{1}$

${ }^{1}$ University of New Mexico Health Sciences Center; ${ }^{2}$ University of New Mexico

ABSTRACT IMPACT: We present a minimally-invasive approach to monitoring heavy metal exposures in geographically disperse populations; this framework may encourage and facilitate greater participation in a broad range of related clinical studies that require biosampling. OBJECTIVES/GOALS: We hypothesize that microneedle array (MA) extraction of interstitial fluid (ISF) will enable minimally-invasive quantitation of heavy metal (HM) exposure. We establish analytical parameters for ICP-MS analysis of HMs in ISF, quantify baseline HM content in ISF vs other fluids, and characterize a mixed HM exposure model. METHODS/STUDY POPULATION: Recent advances in ISF extraction and analysis suggest a minimally-invasive to monitor HM exposure longitudinally in both urban and dispersed communities. ISF can be collected with MAs and is a rich source of disease and exposure biomarkers. To refine analytical methods, Human subjects with no underlying skin disease were recruited into the IRB-approved study. Each subject had blood and urine collected. ISF was also collected using 3D-printed MA-holders. The fluids were analyzed, using ICP-MS, to quantify the levels of uranium $(\mathrm{U})$, cadmium $(\mathrm{Cd})$, vanadium $(\mathrm{V})$, and arsenic (As). Additionally, we analyzed 2,770 subjects from the public NHANES dataset from 2018-2019. Python and Scikit-learn were used to analyze the demographics, survey responses, and metal concentrations for these individuals. RESULTS/ANTICIPATED RESULTS: While several studies have described the surface water and sediment content of toxic metals, determining biological loads remains challenging due to the need to collect blood or urine from a dispersed rural population over time. Our preliminary results suggest similar HM concentrations in ISF, compared with blood in a small unexposed population. Analysis of subjects from the NHANES public datasets suggest similar baseline blood HM concentrations in diverse subject populations with some differences in $\mathrm{Cd}$ depending on smoking and e-cigarette usage. Correlation maps also suggest possible synergy between different metals with cobalt and chromium showing the highest correlation. The initial results from this study have been applied to develop a mixed HM exposure model in rats for further translational testing. DISCUSSION/ SIGNIFICANCE OF FINDINGS: We present a minimally-invasive $\mathrm{HM}$ monitoring approach. Exposure to multiple HMs is suspected to have additive or synergistic harmful health effects. We ultimately envision a wearable microneedle patch that could be mailed to individuals or distributed through community centers, worn for a few hours, and returned to a central laboratory.

76215

\section{Implementation of Proteomics as a Diagnostic tool for Nontuberculous mycobacteria (NTM) Infection}

Nicole Lapinel MD, Jessie Guidry, Mary Varkey MBBS, Manish Rijal, Arnold Zea PhD and Juzar Ali, MD

Louisiana State University Health Sciences Center

ABSTRACT IMPACT: Implementation of proteomics as a diagnostic tool for Nontuberculous mycobacteria (NTM) infection can provide a more accurate, efficient and cost-effective means for effectively diagnosing disease and enacting timely management decisions which can revolutionize patient care. OBJECTIVES/GOALS: Proteomic analysis is a proven diagnostic modality enabling rapid identification of microorganisms. We sought to apply proteomics to detect proteins unique to the most clinically relevant NTM. We then determined whether these unique proteomes could be used to successfully identify NTM species from in vitro cocktail preparations. METHODS/STUDY POPULATION: NTM reference strains for M. avium, m. intracellulare, m.chimaera, m. abscessus abscessus, $\mathrm{m}$. abscessus massiliense and $\mathrm{m}$. abscessus boletti were cultured in vitro and subjected to proteomic analysis using Liquid Chromatography tandem-Mass Spectrometry (LCMS). Tandem Mass Tag (TMT) data acquisition utilized an MS3 approach for data collection using Proteome Discoverer 2.4.

A comparative analysis of the proteome of each of these six species was performed quantitatively using LCMS. The process was repeated for three technical replicates and analyzed using the SEQUEST algorithm. Only high scoring peptides were considered utilizing a false discovery rate (FDR) of $1 \%$. Once species-specific proteins were identified, we validated detection in individual and mixed samples of the six reference strains. RESULTS/ANTICIPATED RESULTS: The proteomic profiling of the six NTM reference strains successfully demonstrated proteins unique to each of the MAC species and MABC subspecies. Proteomic MAC species analysis produced 
between 327 to 2,540 unique peptides for each of the 3 species. MABC proteomic analysis identified between 17-74 unique peptides for each of the 3 subspecies. Fifteen different mixed preparations of MAC and MABC were then subjected to LCMS analysis and compared against the proteome profiles already curated for the six strains. We accurately identified at least one NTM in the majority of the samples (10/15). In three samples (3/15), the NTM was not correctly identified; in two of the samples $(2 / 15)$ we were unable to determine the identity of NTM within the preparation. Further database curation will be performed to hone these results. DISCUSSION/SIGNIFICANCE OF FINDINGS: Proteomic analysis of in vitro reference strains successfully demonstrated protein fingerprints specific to six common disease-causing strains of NTM. Such findings can be used to evaluate clinical samples enabling more efficient diagnostic specificity. Further research will focus on identification of NTM in sputum samples of infected patients.

\section{Precision Medicine}

\section{6}

Patterns and impact of long-term glucocorticoid use on RA patients at risk for major adverse cardiac events Beth I. Wallace ${ }^{1}$, Yuqing $\mathrm{Gao}^{2}$, Punyasha Roul ${ }^{3}$, Shirley CohenMekelberg $^{4}$, Bryant England ${ }^{3}$, Ted Mikuls ${ }^{3}$, Daniel J. Clauw ${ }^{5}$, Rodney Hayward $^{6}$ and Akbar K. Waljee ${ }^{4}$

${ }^{1}$ Center for Clinical Management Research, VA Ann Arbor Healthcare Center and Division of Rheumatology, Department of Internal Medicine, Michigan Medicine; ${ }^{2}$ Center for Clinical Management Research, VA Ann Arbor Healthcare Center; ${ }^{3}$ Division of Rheumatology and Immunology, Department of Internal Medicine, University of Nebraska Medical Center; ${ }^{4}$ Center for Clinical Management Research, VA Ann Arbor Healthcare Center and Division of Gastroenterology, Department of Internal Medicine, Michigan Medicine; ${ }^{5}$ Department of Anesthesiology, Michigan Medicine; ${ }^{6}$ Division of General Medicine, Department of Internal Medicine, Michigan Medicine

ABSTRACT IMPACT: Glucocorticoid steroids are commonly used despite known dose-dependent cardiovascular toxicity, yet little is known about a) how patients with other cardiovascular risk factors use glucocorticoids, and b) how risks of glucocorticoid treatment might vary depending on a patient's baseline cardiovascular risk. OBJECTIVES/GOALS: Up to one-third of RA patients use longterm glucocorticoids (GCs) despite a known, dose-dependent association with increased risk of major adverse cardiovascular events (MACE). We aim to evaluate patterns of GC use among RA patients with other MACE risk factors (i.e. diabetes, smoking), and examine how GC use may potentiate these risk factors. METHODS/STUDY POPULATION: We used claims data from Veterans Health Administration to identify 6,090 RA patients with $\geq 1$ rheumatology clinic visit during 2013-2017. We used logistic regression to evaluate associations between incident MACE between 2013-2018, recent long-term GC use, and 5 MACE risk factors: hypertension, diabetes, hyperlipidemia, smoking, and prior MACE. We included two-way interaction terms between GC use and each risk factor. We used a claims-based algorithm to define MACE as any of acute MI, ischemic stroke, TIA, sudden death, or coronary revascularization, between index date and 12/31/2018. We defined index date as first rheumatology visit after meeting RA diagnostic criteria, and recent long-term GC use as $\geq 90$ days' supply dispensed over 2 years prior to index date. RESULTS/ANTICIPATED RESULTS: Among 2,884 eligible patients, 1,553 (54\%) had MACE risk factors, and 97 (3\%) had prior MACE (Table 1). Overall, $16 \%$ of patients recently used longterm GC, compared to $17 \%$ of patients with MACE risk factors, and $22 \%$ of patients with prior MACE. Incident MACE occurred in 308 (11\%) patients, $24 \%$ of whom had recent long-term GC use. Recent long-term GC use was independently associated with increased incident MACE (Table 2). While no interaction term was statistically significant overall, differences in odds of incident MACE were seen across levels of recent GC use for several risk factors, particularly diabetes (OR 2.10, 95\% CI [0.93-4.77]), tobacco use (OR 2.88, 95\% CI [1.16-7.14]) and prior MACE (OR 2.41, 95\% CI [0.73-7.95]). DISCUSSION/SIGNIFICANCE OF FINDINGS: Long-term GC use is common among RA patients with MACE risk factors. In this cohort, $25 \%$ of patients with incident MACE had recently used longterm GC. Long-term GC use may potentiate effects of comorbidities like diabetes and smoking, disproportionately increasing MACE risk in certain patients.

\section{4}

Fast strain-encoded cardiac magnetic resonance detects immune checkpoint inhibitor associated cardiotoxicity

Jason Cuomo, MD, MA, Elio Ragheb, MD, Attila Feher, MD, PhD, Jennifer M. Kwan, MD, PhD, Steffen Huber, MD, Hamid Mojibian, MD, Dana C. Peters, PhD, Albert Sinusas, MD, * Lauren A. Baldassarre, MD

Yale School of Medicine

ABSTRACT IMPACT: Advanced cardiac magnetic resonance imaging techniques can help to protect cancer patients from cardiotoxicity from immunotherapy with a more sensitive assessment of cardiac function with strain imaging for detection of abnormal cardiac function in the setting of normal left ventricular ejection fraction. OBJECTIVES/GOALS: Immune checkpoint inhibitors (ICI) are associated with fatal cardiotoxicity. Cardiac magnetic resonance (CMR) imaging can assess ICI-associated cardiotoxicity, but the utility of CMR strain imaging is unknown. We present a study of patients with ICI-associated cardiotoxicity evaluated with fast strain-encoded (fast-SENC) CMR. METHODS/STUDY POPULATION: This prospective study was approved by the institutional IRB and informed consent was obtained from 15 patients (5 patients with ICI-associated cardiotoxicity, 10 controls patients) between August 2018 and January 2020. All patients with ICI-associated cardiotoxicity had abnormal troponin values and evidence of cardiotoxicity on T2-weighted and/or delayed enhancement CMR images. All patients underwent standard CMR assessment with steady state free precession cine images, T2-weighted imaging, and delayed gadolinium enhancement imaging. Additionally, freebreathing SENC images were obtained and then processed by a team of blinded cardiovascular imaging specialists using Myostrain software (Morrisville, USA). RESULTS/ANTICIPATED RESULTS: Left ventricular ejection fraction (LVEF) was normal in both groups (i, $\left.{ }^{3} 53 \%\right)$. Global longitudinal LV strain was significantly depressed in the ICI cardiotoxicity group versus controls $(-12.8 \pm 3.2 \%$ vs. -16.6 $\pm 1.9 \%, p=0.028)$. The average global circumferential LV strain was mildly abnormal (defined as strain $>-17$ ) in the ICI cardiotoxicity group and trended towards a higher value compared with controls $(-16.0 \pm 2.6 \%$ vs $-17.8 \pm 1.7 \%, \mathrm{p}=0.103)$. The average number of dysfunctional segments (defined as strain $>-10$ ) was significantly 